\title{
Concepciones alternativas de discontinuidad de la materia, a partir de una exploración documental histórico epistemológica, diseño y aplicación de un instrumento de ideas previas
}

\author{
Jhon J. Valderrama, Julián Vergara y Sandra Isabel Enciso
}

Universidad Distrital Francisco José de Caldas. jhonjairo.valdenrama@gmail.com , jvergara7@gmail.com, sandra.enciso@gmail.com

\section{Resumen}

Se parte de una exploración documental histórico epistemológica del concepto Discontínuidad de la materia, a partir de este se plantearon categorías de análisis para el diseño del instrumento de ideas previas: La materia esta formada por pequeñas partíalas que no podemos ver, Las partículas se encuentran en continuo movimiento, la noción de Vacío, entre otras. Posteriormente se realiza la aplicación a 35 estudiantes de grado 11 del IED Policarpa Salavarieta y el análisis del instrumento por cada pregunta teniendo en cuenta las categorías de análisis.

De lo anterior conduimos que, aunque las estudiantes están por terminar su educación madia vocacional siguen dando explicaciones a los fenómenos a partir de concepciones altemativas macroscópicas, lo cual dificulta el aprendizaje significativo de la química en general.

\section{Palabras claves}

Discontinuidad de la materia, concepciones altemativas, enseñanza - aprendizaje, didáctica de las ciencias, ciencia escolar.

\begin{abstract}
Begin is a documentary exploration of the historical epistemological discontinuity concept of matter, from this raised level of analysis for the design of the instrument prior ideas: Matter is made up of small partides that can not see, partides are continuous movement, the notion of vacuum, among others. Thereafter the application is made to 35 students in grade 11 IED Policarpa Salavarnieta and analysis tool for each question taking into account the categories of analysis.
\end{abstract}

From the above we condude that while students are completing their vocational education still giving explanations to the phenomena from the macroscopic alternative conceptions, which significantly impedes the learning of chemistry in general.

\section{Keywords}

Discontinuity of matter, alternative conceptions, teaching - learning, science teaching, science education.

\section{Introducción}

El estudio de la química permite que los estudiantes comprendan la naturaleza de los diversos fenómenos que afectan su entomo, reconociendo así no sólo el hecho mismo sino su proceso, el mecanismo en el cual el estudiante puede dar respuesta a los diferentes interrogantes está directamente relacionado con el proceso de enseñanza-aprendizaje (desde el conocimiento cotidiano hasta el conocimiento científico escolar), recordando lo planteado por Ausubel "conózcase 
lo que el estudiante sabe y enséñese en consecuencia" es indispensable plantear una estrategia por medio de la cual se puedan reconocer las concepciones altemativas de los estudiantes acerca de los conceptos estructurantes de la química Discontinuidad de la Materia, Cambio Químico, Cuantificación de Relaciones los auales se enumeran en diferentes investigaciones (Pozo, 1991, Garća, Mosquera y Mora 2003).

El concepto en el cual se centra esta investigación es el de discontinuidad de la materia, debido a que varios autores (Villaveces 2000, Garáa, Mosquera y Mora 2003, Pozo 1991, Uorens 1988) señalan la baja comprensión de los conceptos que este envuelve; noción de vació, composición corpuscular de la materia, movimiento intrínsico de la materia, ademas el concepto permite al estudiante dar una explicación de los fenómenos macroscópicos partiendo de lo que sucede a nivel microscópico, esto facilitará la resolución de cualquier problemática tanto a nivel científico escolar (Mercé Izquierdo 1997) como cotidiano.

Por lo anteriormente descrito se establece que del concepto de discontinuidad de la materia se puede partir para la enseñanza de la química, razón por la aual se desarrollo esta investigación en la cual se construye un instrumento para categorizar las concepciones alternativas presentes en estudiantes de grado once del colegio IED Policarpa Salavarnieta, en cuanto al nivel de aprendizaje del concepto discontinuidad de la materia.

\section{Objetivos}

Diseñar, aplicar y categorizar las concepciones altemativas de las estudiantes de grado 11 del I.E.D. Policarpa Salavarieta acerca del concepto de discontinuidad de la materia.

\section{Específicos}

- Realizar un análisis documental de carácter; histórico, didáctico y epistemológico del concepto discontinuidad de la materia.

- Construir las categońas de análisis de las concepciones altemativas de las estudiantes sobre el concepto de discontinuidad de la materia, a partir del marco teórico planteado para éste.

- Diseñar, validar, estandarizar y aplicar el instrumento como una prueba objetiva.

- Analizar las concepciones recogidas a partir de la aplicación del instrumento de las estudiantes acerca de la concepción de la discontinuidad de la materia.

\section{Metodología}

Se parte de una exploración documental (Fase exploratoria) histórico epistemológico del concepto Discontinuidad de la materia, el aul no se encuentra explíatamente en el tratamiento histórico de la química, sino en los estudios didácticos, desde la historia este concepto surge de la integración de varias teorías, como lo son, la composición corpuscular de la materia que se desarrolla desde la concepción atomista planteada inicialmente por Demócrito y Leucipo con grandes aportes de Epicuro, quienes establecieron que la materia estaba conformada por unas partículas indivisibles (Villaveces 2000); aunque esta idea no fue de gran aceptación por los filósofos de la época quienes seguían la concepción sustancialista, la cual se incorporó en la estructura de diversas teorías, ésta solo pudo empezarse a superar a partir de las concepciones planteadas por Boyle en su publicación El químico escéptico y las posteriores teorías atómicas.

Por otra parte, otro de los conceptos integrados en la discontinuidad es el movimiento intrínseco de las partículas que constituyen la materia, planteado por Galileo y los Racionalistas en el S. XVII quienes tomando teorías de Heron dotaron a las partículas de movimiento para explicar las propiedades de la materia siendo esta la base para la "filosofía mecánica". 
Por ultimo la noción de vacío fue incorporada por Gassendi enmarcado en la química Mecánica, al aceptar las pruebas experimentales expuestas por Torricelli en donde prueba la existencia del vacío, Gassendi estimaba que los átomos se movían en este vacío.

Además de los tres conceptos inmersos en la discontinuidad de la materia, consideramos de gran importancia la concepción de conservación de la masa que fue establecida por primera vez de modo eficaz por Lavoisier quien al estudiar la fermentación del azúcar postula que nada se crea, que en toda operación existe la misma cantidad de materia antes y después de la operación. (Leicester 1967).

El diseño metodológico tiene la siguiente estructura:

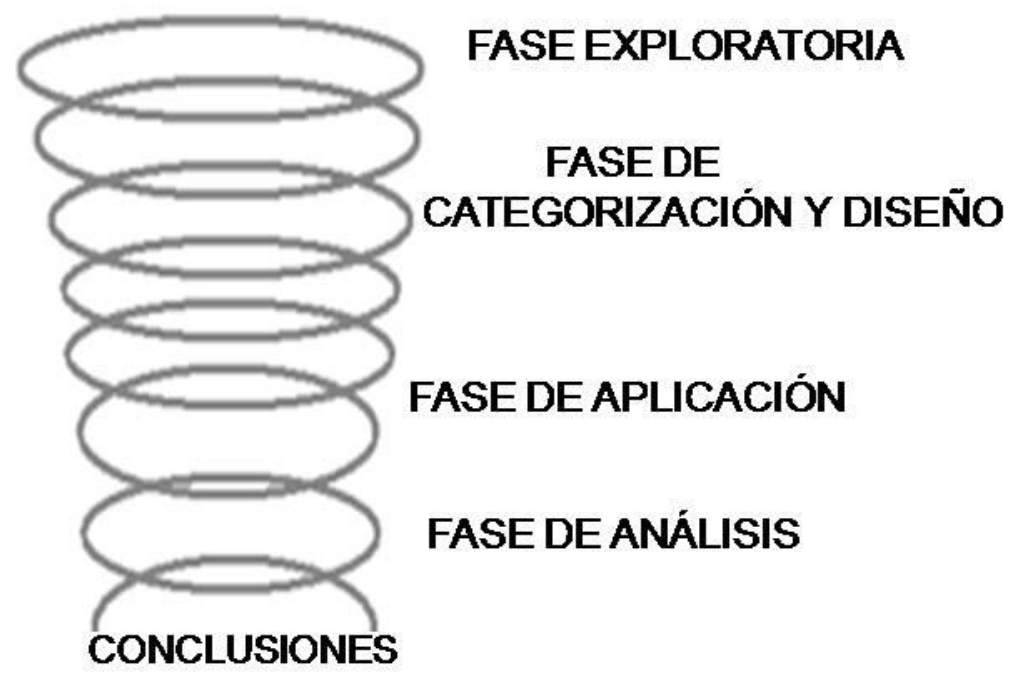

La cual se desarrolla por fases en forma de espiral, lo que permite retomar los elementos explorados en las diferentes fases.

Fase Exploratoria (Descrita anteriormente)

Fase de Categorización y Diseño

Las categorías fueron establecidas a partir de la fase de exploración documental, teniendo en cuenta las teorías que integran el concepto de Discontinuidad de la materia, además de cambios de estado y conservación de la materia, con estos, se diseño un instrumento que consta de 10 que pasaron por un proceso de validación y estandarización para dar confiabilidad al instrumento, las categorías de análisis y objetivos de éstas se pueden observar en la tabla de especificaciones presentada en los resultados (Ver tabla 1 y 2 )

Fase de Aplicación

La muestra total en la aual se aplico el instrumentó fue de 35 estudiantes de grado once del IED Policarpa Salavarrieta, con edades entre los 15 y 17 años.

El tiempo empleado por las estudiantes para la resolución total del instrumento fue de una hora; adarando que la entrega de éste comenzó pasados los cuarenta y cinco minutos.

Fase de Análisis

Se recolectaron y tabularon los resultados para su posterior análisis teniendo en cuenta las categorías de análisis anteriormente planteadas. 


\section{Resultados}

El instrumento contó con 10 preguntas, 3 preguntas de afirmación y negación, 1 pregunta para completar, 1 pregunta abierta y 5 preguntas de selección múltiple.

En la tabla que se presentan a continuación se describen los objetivos, categorías de análisis y el numeral en el cual se valoran cada una de éstas.

\begin{tabular}{|c|c|c|c|c|}
\hline \multirow{2}{*}{ CATECORÍAS DE ANÁLISIS } & \multicolumn{4}{|c|}{ OBJETIVOS } \\
\hline & Conocimiento & Comprensión & Aplicación & Argumentación \\
\hline $\begin{array}{l}\text { Características de los estados } \\
\text { de la materia }\end{array}$ & $\mathbf{x}$ & & $\mathbf{x}$ & $\mathbf{x}$ \\
\hline $\begin{array}{l}\text { La materia esta formada por } \\
\text { pequeñas partículas que no } \\
\text { podemos ver "átomo" }\end{array}$ & $\mathbf{x}$ & $\mathbf{x}$ & $\mathbf{x}$ & $\mathbf{x}$ \\
\hline $\begin{array}{l}\text { Las partículas se encuentran } \\
\text { en continuo movimiento }\end{array}$ & $\mathbf{x}$ & $\mathbf{x}$ & $\mathbf{x}$ & $\mathbf{x}$ \\
\hline $\begin{array}{l}\text { Entre las partículas no hay } \\
\text { absolutamente nada "Vacio" }\end{array}$ & & $\mathbf{x}$ & $\mathbf{x}$ & $\mathbf{x}$ \\
\hline $\begin{array}{l}\text { Diferencias entre los estados } \\
\text { de la materia "solidó, liquido y } \\
\text { gaseoso" y cambios de estado. }\end{array}$ & $\mathbf{x}$ & $\mathbf{x}$ & $\mathbf{x}$ & $\mathbf{x}$ \\
\hline
\end{tabular}

Tabla 1. Tablas de especificaciones e instrumento didáctico.

Qué se busca con cada pregunta de:

Con las preguntas se busca indagar sobre las concepciones que tienen los estudiantes acerca

> Características de los estados de la materia, nivel macroscópico: 1-2

> La materia esta formada de partículas diminutas, nivel microscópico: 3-4

> Indagan sobre la corpuscularidad de la materia, la posibilidad de que existan espacios vacíos entre las partículas, y el movimiento que tienen estas: 5-8

$>$ Como aplican el modelo discontinuo en los cambios de estado: 9-10

Teniendo en cuenta expuesto anteriormente, el instrumento fue realizado de esta forma ya que el concepto de discontinuidad tiene cuatro aspectos que se deben comprender para llegar a este:

1. La materia esta formada por pequeñas partículas que no podemos ver "átomo"

2. Las partículas se encuentran en continuo movimiento

3. Entre las partículas no hay absolutamente nada "Vacío"

4. Conservación de la materia.

Los cuales están ordenados desde el más fácil de comprender por los estudiantes (1), ya que le da explicaciones a fenómenos macroscópicos con los que se enfrentan a diario de una manera microscópica, obteniendo la noción de materia conformada por pequeñas partículas.

Con esta noción es mucho más fácil explicar el movimiento continuo de estas partículas con el fenómeno de la difusión.

Por ultimo se podría llegar al concepto de vacío teniendo en cuenta que cuando ocurren fenómenos como la dilatación de los gases nunca se introduce más gas pero este aumenta su volumen. 
Para finalizar obtenemos la aplicación y argumentación de los anteriores ítems con la explicación de los cambios de la materia.

Además con la argumentación nos daremos cuenta si sus conceptos son significativos o memorísticos

Teniendo en cuenta estos criterios para la categorización de las concepciones alternativas de las estudiantes del I.E.D. Policarpa Salavarrieta, se establecen los siguientes resultados acerca del concepto de discontinuidad para cada una de las categońas.

1. Diferencias entre los estados de la materia: "sólido, líquido y gaseoso"

Las concepciones evaluadas sobre sólidos, dieron una respuesta positiva por parte de los estudiantes, evidenciándose la comprensión del aspecto macroscópico.

Para los líquidos, existen concepciones altemativas en donde relacionan directamente el cambio de la masa con el del volumen y este ultimo con el cambio de forma.

Para los gases en su mayoría los estudiantes tienen la concepción de que los gases no conservan su masa.

En general las estudiantes tienen daro cuales son los estados de la materia, pero en su diferenciación se centran en las características perceptibles (mundo Macro).

2. La materia esta formada de partículas diminutas.

Las estudiantes, manejan la concepción de que la materia es continua; que los animales y vegetales están formados por células, y los minerales y gases por partículas sin diferenciar átomo de molécula

3. Entre las partículas no hay absolutamente nada "Vacío"

En cuanto a la noción de vacio, esta no se ve representada por las estudiantes para el estado solido y liquido teniendo una concepción continua de la materia, al trabajar con gases la concepción acerca de vacio es un poco mas dara, ya que algunas estudiantes representan los gases como partíaulas.

4. Las partículas se encuentran en continuo movimiento

Ellos le atribuyen a las partículas características de quietud y movimiento dependiendo de la visión macroscópica que tengan de los estados de agregación; donde para los líquidos y sólidos creen la necesidad de un agente externo para provocar su movimiento, a diferencia de los gases que no lo necesitan, siempre están en continuo movimiento.

5. La materia no se crea ni se destruye, solo se transforma.

En las representaciones corpusaulares de los gases al comprimirse se disminuye el espacio entre las partículas, aunque para la mayoría de estudiantes la compresión y expansión de los gases se da por aparición y desaparición de estas.

En la representación grafica realizada por las estudiantes para cambios de estado no hay relación entre la cantidad de partículas que están presentes en el líquido con las representadas para el gas, es decir, la materia inicial no es igual a la final.

\section{Conclusiones}

Das ideas previas que los estudiantes tienen acerca de las características de los estados de agregación de la materia son a nivel microscópico, esto hace que al diferenciar un estado de otro comentan errores conceptuales como: no conservan la materia, atribuyen a un cambio físico un cambio químico, transfieren las propiedades de los gases a los líquidos, manteniendo 
estables las de los sólidos, ya que son estos últimos para ellos los mas consistentes, por esta razón conservaran todas sus características (forma, volumen y materia).

> La gran mayoría de estudiantes le otorgan a la materia una composición continua, pues se basan en lo perceptible, tangible y aquellas que logran entender la teoría corpuscular no diferencian entre átomo y molécula.

$>$ Las estudiantes, a pesar de su concepción continúa de materia, tienden a representar gráficamente un espacio entre las partículas solamente para los gases, ya que esta propiedad no la pueden trasponer con gran facilidad a líquidos y sólidos.

$>$ En la representación grafica realizada por las estudiantes para cambios de estado no hay relación entre la cantidad de partículas que están presentes en el líquido con las representadas para el gas, es decir, la materia inicial no es igual a la final. No hay conservación de la metería.

$>\quad$ Las estudiantes atribuyen a las partículas características de quietud y movimiento dependiendo de la visión macroscópica que tengan de los estados de agregación; donde para los líquidos y sólidos creen la necesidad de un agente externo para provocar su movimiento, a diferencia de los gases.

- Las estudiantes debido a la visión macroscópica que tienen de los cambios de estado, con frecuencia confunden un cambio químico con un cambio físico.

> aunque las estudiantes están por terminar su educación madia vocacional y ya tienen una formación en química, esta formación no es nada conceptual sino memorística, además que siguen dando explicaciones a los fenómenos a partir de concepciones altemativas macroscópicas, lo cual dificulta el aprendizaje significativo de la química en general.

\section{Bibliografía}

García, Mosquera y Mora (2003). Conceptos Fundamentales de la química y su relación con el desarrollo profesional del profesorado. Bogotá

Leicester H. (1967). Panorama histórico de la química. Madrid

Lorens, J. A. (1988). La concepción corpusaular de la materia. Obstáculos epistemológicos y problemas de aprendizaje. Investigación en la escuela, 4, 33-48

Pozo, J.I., Gómez crespo, M. Limón, m. Sanz, a. (1991). Procesos cognítivos en la comprensión de la ciencia: las ideas de los adolescentes sobre la química Madrid: CIDE.

Sanmarti, N. e Izquierdo, M. (1997) Reflexiones en torno a un modelo de ciencia escolar. Revista Investigación en la Escuela, 32, 51-62

Villaveces C. (2000). Química y Epistemología, una Relación Equivoca Revista colombiana de filosofía de la ciencia. Vol 1, № 2 y 3. 9-25. Bogotá 